\title{
Perfil cognitivo e funcional de idosos moradores de uma instituição de longa permanência para idosos
}

\author{
Renata Souza Mendes, Marcia Maria Pires Camargo Novelli \\ Universidade Federal de São Paulo - UNIFESP, São Paulo, SP, Brasil.
}

\begin{abstract}
Resumo: Introdução: O Brasil é um dos países em desenvolvimento onde a população de idosos mais cresce. Nesta fase da vida, estão presentes alterações, como cognitivas e funcionais, que têm grande influência na vida do indivíduo e que podem levar à institucionalização. Objetivo: Identificar o perfil cognitivo e funcional de idosos de uma ILPI em Santos-SP e verificar correlações entre as variáveis. Métodos: Utilizou-se avaliação cognitiva e funcional, além de questionário de variáveis sociodemográficas. Participaram do estudo 15 idosos. Resultados: $100 \%$ dos idosos avaliados apresentaram déficits cognitivos e funcionais. Em relação aos déficits cognitivos, foi identificado prejuízo, principalmente, nos domínios de Construção Visomotora e Operações de Pensamento. O uso da mediação melhorou o desempenho dos idosos, significativamente, nos domínios de Percep̧̧ão Visual, Percepção Espacial, Práxis e Construção Visomotora. Os idosos apresentaram 54\% de independência nas AVDs, sendo 66,27\% de independência nas ABVDs (principalmente alimentação e continência) e 52,18\% nas AIVDs (principalmente uso do telefone). Houve associação entre cognição e funcionalidade ( $\mathrm{r}=0,57 ; \mathrm{p}<0,05)$. Conclusão: Os idosos avaliados mostraram prejuízos cognitivos e funcionais, com forte correlação; sugere-se avaliação periódica para que novas ações sejam propostas para melhora e manutenção funcional dos idosos.
\end{abstract}

Palavras-chave: Idosos, Institucionalização, Avaliação Cognitiva, Avaliação Funcional.

\section{Cognitive and functional profile of residents of a long term institution for elderly}

\begin{abstract}
Introduction: Brazil is one of the developing countries where the elderly population grows more. At this stage of life there are cognitive and functional changes with great influence on the person's life, which may lead to institutionalization. Objective: To identify the cognitive and functional profile of elderly living in a long term institution for elderly in Santos/SP and verify correlations. Methods: We used cognitive and functional assessment and, a questionnaire of socio-demographic variables. The study included 15 elderly individuals. Results: All respondents had cognitive and functional deficits. Regarding cognitive deficits we identified impairments, mainly in the Visual motor Construction and Thinking Operations. The use of mediation improved the performance of the elderly, most significantly, in Visual Perception, Space Perception, Praxis and Visual motor Construction. The elderly showed $54 \%$ of ADL independence, which showed $66.27 \%$ of BADL independence (especially feeding and continence) and $52.18 \%$ in IADL (especially use of the phone). There was an association between cognition and function $(r=0.57, p<.05)$. Conclusion: The elderly assessed exhibit cognitive and functional impairments, it is suggested periodic assessment so that new actions are proposed for improvement and maintenance of their functionality.
\end{abstract}

Keywords: Elderly, Institutionalization, Cognitive Assessment, Functional Assessment.

\footnotetext{
Autor para correspondência: Marcia Maria Pires Camargo Novelli, Departamento de Gestão e Cuidados em Saúde, Universidade Federal de São Paulo, Campus Baixada Santista, Rua Silva Jardim, 136, Vila Mathias, CEP 11015-020, Santos, SP, Brasil, e-mail: mnovelli@uol.com.br Recebido em Nov. 8, 2013; $1^{\text {a }}$ Revisão em Dez. 11, 2014; Aceito em Fev. 23, 2015.
} 


\section{Introdução}

\subsection{Envelhecimento}

O Brasil é um dos países em desenvolvimento onde a população de idosos mais cresce. Estima-se que o número de idosos, com 60 anos ou mais, será de 30 milhóes para 2025, atingindo, então, o sexto lugar no ranking mundial (VAGETTI; WEINHEIMER; OLIVEIRA, 2007). De acordo com o censo 2010 (INSTITUTO..., 2010), os indivíduos acima de 60 anos correspondem a 10,7\% da população total brasileira.

De forma geral, o Brasil passa por um processo de transição demográfica, com uma mudança no padráo de causas de morte, de um perfil de doenças infectocontagiosas para um perfil de doenças crônico-degenerativas, que podem ter um grande impacto na funcionalidade e na mortalidade de idosos (PARENTE et al., 2006).

Embora a grande maioria dos idosos tenha condiçôes de viver e se manter sozinhos ou com ajuda de familiares, existe uma parcela com uma crescente demanda de institucionalização, fato este que tem grande influência na vida de um idoso (VAGETTI; WEINHEIMER; OLIVEIRA, 2007).

O envelhecimento humano passa por várias alteraçôes, que podem comprometer diversas capacidades nos idosos. Os déficits cognitivos, como uma destas alterações, causam impactos sobre cada aspecto da vida de uma pessoa e podem criar dificuldades de desempenho em todas as áreas de ocupaçáo, como Atividades Básicas de Vida Diária (ABVD), Atividades Instrumentais de Vida Diária (AIVD), Lazer e Trabalho, de forma que a capacidade funcional do idoso pode ser afetada, gerando incapacidade. Tal situação pode levar muitos idosos a serem institucionalizados, gerando neles uma disfunção ocupacional pela desestruturação ou mudança da rotina diária, além da incapacidade funcional que já apresentavam (GRIEVE; GNANASEKARAN, 2010; PEDRETTI; EARLY, 2004).

\subsection{Cognição, funcionalidade e terapia ocupacional}

A cognição descreve toda a esfera do funcionamento mental, que inclui habilidade de sentir, pensar, perceber, lembrar, raciocinar, formar pensamentos complexos e responder aos estímulos externos (VIEIRA; KOENIG, 2002).

Existe consenso entre os pesquisadores da área da cognição de que o processo de envelhecimento acarreta um declínio normal, podendo apresentar-se desde os anos da meia-idade, e tornando-se mais comum depois dos 70 anos. Sabe-se, também, que há uma variabilidade em relação aos domínios da cognição que declinam, em relação ao ritmo e às consequências desse declínio no envelhecimento (NERI; NERI, 2011).

Geralmente, os idosos se queixam de dificuldades com a memória e outras capacidades cognitivas, principalmente ao comparar seu desempenho atual com o do passado. Tais alteraçóes cognitivas são comuns entre os idosos e só adquirem significado quando afetam o desempenho das atividades diárias do indivíduo, prejudicando-as (CANINEU; STELLA; SAMARA, 2011).

Os déficits cognitivos em um idoso são, muitas vezes, a origem de problemas funcionais, fazendo com que haja perda ou dificuldade para adquirir ou manter as capacidades e habilidades. Com isso, ocorrem mudanças nos contextos de vida do indivíduo (sociais, econômicos e de vida diária) (GRIEVE; GNANASEKARAN, 2010).

De acordo com a Classificação Internacional de Funcionalidade (CIF), o termo funcionalidade abrange as funçóes e estruturas do corpo; atividade e participação social, e fatores ambientais (FARIAS; BUCHALLA, 2005).

A funcionalidade diz respeito ao uso das estruturas e funçóes do corpo para o desempenho de atividades de maneira a satisfazer necessidades e desejos de vida, utilizando recursos pessoais em determinados ambientes e culturas. Refere-se à capacidade de a pessoa cuidar de si mesma e de desempenhar tarefas e papéis sociais (PERRACINI, 2011).

No raciocínio clínico da Terapia Ocupacional, as perspectivas supramencionadas são consideradas, ou seja, o desempenho nas atividades (desempenho real da pessoa nos contextos de seu ambiente normal) - desempenho ocupacional - vai ser enfatizado. Esse desempenho engloba várias atividades: $\mathrm{ABVD}, \mathrm{AIVD}$, trabalho e lazer (NEISTADT; CREPEAU, 2002; KIELHOFNER; BURKE; IGI, 1991; BARRETO; TIRADO, 2002).

O desenvolvimento, o desempenho e a manutenção do desempenho ocupacional sáo influenciados por elementos intrapessoais (aspectos temporais dos contextos do desempenho, bem como fatores genéticos, neurofisiológicos e patológicos) e extrapessoais (ambiente físico, elementos sociais, culturais e familiares). Neste contexto, o papel do Terapeuta Ocupacional é facilitar tanto o equilíbrio do desempenho ocupacional, como sua otimizaçáo. $\mathrm{Na}$ Gerontologia, o Terapeuta Ocupacional estimula 
as atividades que podem ser realizadas dentro das capacidades funcionais do individuo; dá estímulos de orientação espacial e temporal associadas ao contexto e à realidade do individuo; dá estímulos de memória e atenção, entre outros (PEDRETTI; EARLY, 2004).

Atualmente, há uma mudança de paradigma, quando se reflete sobre o construto funcionalidade e envelhecimento, na medida em que se incluem na determinação da funcionalidade e incapacidade, aspectos relacionados ao contexto do ambiente físico e social, a diferentes percepçóes frente à incapacidade, a dependência e fragilidade na velhice (PERRACINI, 2011; FARIAS; BUCHALLA, 2005).

\subsection{Institucionalização}

O processo de transiçẫo demográfica, como também fatores sociais e de saúde, que vem ocorrendo no Brasil, exige transformaçóes sociais e aumenta a demanda por ILPI frente a incapacidade e dependência dos idosos. As ILPI são uma alternativa de assistência e devem assegurar qualidade de vida e satisfação aos idosos e famílias atendidas (DAVIM et al., 2004).

No Brasil, a origem das ILPI está relacionada aos asilos, inicialmente dirigidos à população carente que necessitava de abrigo, frutos da caridade cristá. Hoje em dia, essas instituiçóes passaram a cuidar de idosos com muitas necessidades, tendo em vista as perdas funcionais, cognitivas, entre outras, integrando-se então à assistência à saúde (CAMARANO; KANSO, 2010).

Os principais motivos de internação dos idosos nas ILPI são doenças físicas e mentais, assim como abandono e miséria (DAVIM et al., 2004). Segundo Born e Boechat (2011), alguns outros aspectos são apontados como fatores de risco para a institucionalização, como síndrome da imobilidade, problemas físicos, depressão, demência, alta hospitalar recente, incontinência, ser do sexo feminino ou viúvo recente, morar sozinho e isolamento social (falta de apoios sociais).

As ILPIs visam a promover e melhorar a qualidade de vida dos idosos, e minimizar as inevitáveis restriçôes que a vida na instituição pode acarretar; assim, buscam manter a autonomia e a independência do idoso, respeitar a manutenção dos papéis sociais, garantir acesso ao melhor cuidado de saúde e proporcionar cuidado integral (BORN; BOECHAT, 2011).

Estudos foram realizados em diferentes cidades do país, com o objetivo de caracterizar populaçóes idosas em ILPI quanto à sua capacidade cognitiva e funcional, e verificar possíveis correlaçóes, bem como comparar o desempenho dos idosos, por meio de avaliaçôes específicas.

Em estudo realizado por Converso e Iartelli (2007), com 115 idosos moradores de três ILPIs, em Presidente Prudente-SP, encontrou-se que $76,72 \%$ dos idosos apresentaram déficits cognitivos e que $76,65 \%$ dos idosos apresentaram bom estado funcional e/ou independência para as AVDs. Outro estudo (GONTIJO, 2010), com idosos de ILPI da cidade de Divinópolis-MG, mostrou que 44,8\% dos idosos avaliados apresentaram déficits cognitivos e que, em relação à capacidade funcional, 76,10\% dos idosos foram considerados independentes ou semidependentes para a realização das AVDs. Tais estudos não especificavam quais domínios cognitivos e quais atividades funcionais estavam comprometidas.

O enfoque do presente estudo foi avaliar os aspectos cognitivos e funcionais de idosos moradores de uma ILPI na cidade de Santos-SP, e verificar possíveis associaçóes entre essas variáveis. Cabe ressaltar que, em relaçáo à funcionalidade dos idosos, este estudo teve como um dos objetivos avaliar também o desempenho nas AIVDs, e não só as ABVDs, além de identificar o impacto dos domínios cognitivos sobre a funcionalidade, o que não foi analisado por estudos anteriores.

\section{Métodos}

Este estudo foi realizado na perspectiva da abordagem quantitativa, sendo uma pesquisa descritiva e de corte transversal. O projeto e os Termos de Consentimento Livre e Esclarecido para o idoso e para o cuidador foram encaminhados e aprovados pelo Comitê de Ética da Universidade Federal de São Paulo sob o n.o 222/11 para, assim, se iniciar a coleta de dados.

\subsection{População}

Os critérios para inclusão dos idosos no estudo foram: residir na instituição, ter mais de 60 anos, não apresentar déficit de linguagem e comunicação que comprometesse a participação no processo de avaliação, não ter problemas de visão e audição que não pudessem ser corrigidos e consentir na sua participação no estudo, assinando o Termo de Consentimento Livre e Esclarecido.

A partir dos critérios de inclusão, foram elegíveis 18 dos 32 idosos moradores da instituição parceira. Dos 18 idosos selecionados, três não puderam participar da avaliação por apresentarem alteraçóes 
de comportamento, que impediram a aplicação e/ou a continuidade do processo de avaliação. A amostra final para análise das informaçóes foi de 15 idosos.

As avaliaçóes foram realizadas durante o período de fevereiro a julho de 2012, ocorrendo, principalmente, no período da tarde, nas dependências da instituição.

\subsection{Instrumentos de avaliação}

Para mapear os dados sociodemográficos, foi utilizado um questionário semiestruturado com informações, como nome, idade, tempo de institucionalização, escolaridade e diagnóstico. Esses dados, especificamente, foram obtidos a partir dos próprios idosos ou a partir dos prontuários clínicos.

Para avaliar a cognição e funcionalidade dos idosos, foram utilizados, respectivamente, os instrumentos Avaliação Cognitiva Dinâmica de Terapia Ocupacional de Loewenstein para a população geriátrica (DLOTCA-G) e Escala de Avaliação de Incapacidade na Demência (DAD).

A bateria DLOTCA-G (KATZ; AVERBUCH; EREZ, 2012) é um instrumento de avaliação cognitiva para idosos, específico da área de Terapia Ocupacional. Tem como objetivo avaliar capacidades cognitivas em oito áreas: Orientação, Percepção Visual e Espacial, Práxis, Organização Visuomotora, Operaçóes de Pensamento, Memória e Consciência, assim como mensurar o potencial de aprendizado e reconhecer estratégias de pensamento pelo uso de avaliação dinâmica.

Por meio da bateria DLOTCA-G, é possível identificar o perfil cognitivo estático e dinâmico do idoso. $\mathrm{Na}$ avaliação estática, o perfil cognitivo do idoso é avaliado sem qualquer tipo de interferência do examinador. $\mathrm{Na}$ avaliação dinâmica, o perfil cognitivo é estabelecido a partir de interferências (mediações) do examinador.

Para a avaliação dinâmica, o examinador utiliza quatro ou cinco opçóes de mediaçóes em cada subteste, em cinco domínios: Percepção Visual, Percepção Espacial, Práxis, Construção Visuomotora e Operaçóes de Pensamento. Essas mediaçóes são um sistema graduado de pistas (gerais para específicas) que o examinador utiliza para modificar a tarefa e, através disto, entender o tipo de informação que é necessária para que o indivíduo complete a tarefa da melhor maneira.

Para os domínios de Construção Visuomotora e Operaçôes de Pensamento, o tempo de desempenho é medido para cada subteste, com um máximo de dois minutos antes que a mediação se inicie. A pontuação consiste em três componentes para cada subteste: pontuação base pré-mediação (pontuaçôes mais altas indicam melhor desempenho), a pontuação de mediação $(1=$ pouca mediação e $5=$ muita mediação) e pontuação pós-mediação (pontuaçôes mais altas indicam melhor desempenho).

A Escala de Avaliação de Incapacidade na Demência (DAD) é uma escala que mede o desempenho nas AVDs na população idosa com déficits cognitivos (BAHIA et al., 2010). Esta escala tem como objetivos quantificar as capacidades funcionais nas AVDs para indivíduos com déficits cognitivos; qualificar as dimensóes cognitivas das incapacidades nas AVDs, examinando as ABVDs e AIVDs em relação a funçóes executivas, permitindo a identificação de áreas problemas, como iniciativa, planejamento e organização, e realizaçáo efetiva.

O instrumento inclui a avaliação de atividades básicas de vida diária (importantes para o autocuidado), atividades instrumentais de vida diária (importantes para a manutenção em um ambiente específico) e de lazer. É importante lembrar que esta avaliação é realizada através de entrevista com o cuidador (BAHIA et al., 2010).

Algumas das atividades avaliadas pelo DAD (preparar as refeiçóes, sair sozinho da instituição, administrar as finanças e correspondências, administrar as medicações e ficar sozinho na casa) não fazem parte da rotina dos idosos moradores desta ILPI. Desta maneira, as questóes da escala relacionadas a estes itens foram mapeadas junto aos cuidadores formais, no sentido de entender se, fora do contexto da instituição, estes idosos teriam capacidade para desempenhar tais atividades.

\subsection{Análise dos dados}

Foram realizadas análises descritivas (médias e desvio padrão) das variáveis sociodemográficas e dos dados de caracterizaçáo das amostras, utilizando-se os instrumentos de avaliaçáo selecionados. Foi utilizado o Coeficiente de Correlação de Pearson para correlacionar as pontuaçóes totais das avaliações e o Coeficiente de Correlaçáo de Spearman, para correlacionar os itens e domínios das avaliações. O grau de significância considerado foi de $\mathrm{p}<0,05$.

\section{Resultados}

As variáveis sociodemográficas da amostra e os dados da aplicaçáo dos instrumentos sáo apresentados na Tabela 1.

A média de idade dos idosos foi de 81,13 anos e a de escolaridade foi de 7,2 anos. Em relaçáo ao tempo de institucionalização, a média de tempo foi de 10,46 meses. Em relação ao tempo de aplicação da bateria DLOTCA-G, a média de tempo gasto 
Tabela 1. Variáveis sociodemográficas da amostra e aplicação dos instrumentos.

\begin{tabular}{lcc}
\hline \multicolumn{1}{c}{ Variáveis } & $\begin{array}{c}\text { Idosos } \\
(\mathbf{n = 1 5})\end{array}$ & $\begin{array}{c}\text { Desvio } \\
\text { Padrão (Dp) }\end{array}$ \\
\cline { 2 - 3 } & Média & $\pm 10,35$ \\
\hline Idade & 81,13 & $\pm 4,17$ \\
Escolaridade $^{\mathrm{a}}$ & 7,20 & ---- \\
Gênero (feminino) $^{\text {Tempo de }}$ & $13^{\mathrm{b}}(86,66 \%)$ & $\pm 7,18$ \\
Temstitucionalização $^{\mathrm{c}}$ & 10,46 & \\
Tempo de avaliação $^{\mathrm{d}}$ & 95,60 & $\pm 24,23$ \\
(DLOTCA-G) $_{\text {DLOTCA-G (0-26) }}$ & 16,84 & $\pm 3,85$ \\
DAD (0-100\%) & & \\
\hline
\end{tabular}

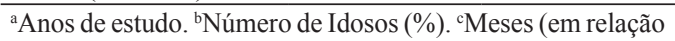
ao período da coleta de dados). ${ }^{\mathrm{d}}$ Minutos.

com a aplicação foi de 95,6 minutos, sendo que, dos 15 idosos avaliados, 12 (80\%) necessitaram de duas sessôes para concluir a avaliação.

A Tabela 2 apresenta as médias da pontuação pré-mediação (estática) por domínio da bateria DLOTCA-G.

Os idosos apresentaram melhor desempenho nas provas de Percepção Espacial e Percepção Visual. Os piores desempenhos ocorreram nas provas de Construção Visuomotora e Operações de Pensamento.

A Figura 1 apresenta o grau de independência em relação às atividades funcionais realizadas pelos idosos.

Os idosos apresentaram maior independência nas atividades de alimentação $(88,89 \%)$, seguidas das atividades de continência (70\%), que fazem parte das ABVDs. As atividades de lazer e atividades de casa $(37,33 \%)$, e o uso do telefone $(38,18 \%)$ apresentaram menor independência (estas fazem parte das AIVDs).

A Figura 2 apresenta o grau de independência em cada uma das atividades que os idosos não realizam por conta da rotina institucional, em porcentagem (\%).

Os idosos apresentariam, segundo o relato dos cuidadores, 54\% de independência nas atividades que não realizam atualmente por conta da rotina e das normas da instituição, sendo que em todas as atividades apresentadas, exceto sair sozinhos de casa, os idosos apresentaram capacidade para executá-las, segundo o relato dos cuidadores.

A Tabela 3 apresenta a correlaçáo entre a avaliação cognitiva e funcional, antes e após a intervenção do examinador com o uso da mediação.

Foram encontradas correlaçôes significativas entre a cognição e a funcionalidade, antes e depois da interferência do examinador.
Tabela 2. Médias da pontuação estática por domínio na DLOTCA-G.

\begin{tabular}{lc}
\hline \multicolumn{1}{c}{ Domínios } & Média \\
\hline Orientação (0-2) & 1,20 \\
Consciência (1-3) & 1,98 \\
Percepção Visual (1-4) & 3,26 \\
Percepção Espacial (0-1) & 0,83 \\
Práxis (0-2) & 1,55 \\
Construção Visuomotora (1-5) & 2,44 \\
Operações de Pensamento (1-5) & 2,53 \\
Memória (1-4) & 3,02 \\
\hline
\end{tabular}

Tabela 3. Correlações entre as pontuações totais da DAD e DLOTCA-G - avaliação estática e dinâmica.

\begin{tabular}{lcc}
\hline & DLOTCA-G & DAD \\
\hline $\begin{array}{l}\text { Avaliação Estática } \\
\text { DLOTCA-G }\end{array}$ & 1 & $0,57^{*}$ \\
DAD & & \\
$\begin{array}{l}\text { Avaliação } \\
\text { Dinâmica }\end{array}$ & $0,57^{*}$ & 1 \\
DLOTCA-G & & \\
DAD & 1 & $0,58^{*}$ \\
\hline
\end{tabular}

${ }^{*} \mathrm{p}<0,05$.

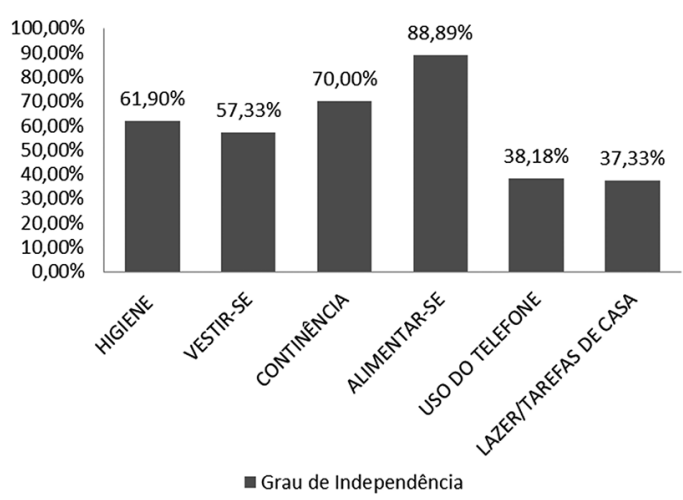

Figura 1. Grau de independência em relação às atividades funcionais realizadas pelos idosos.

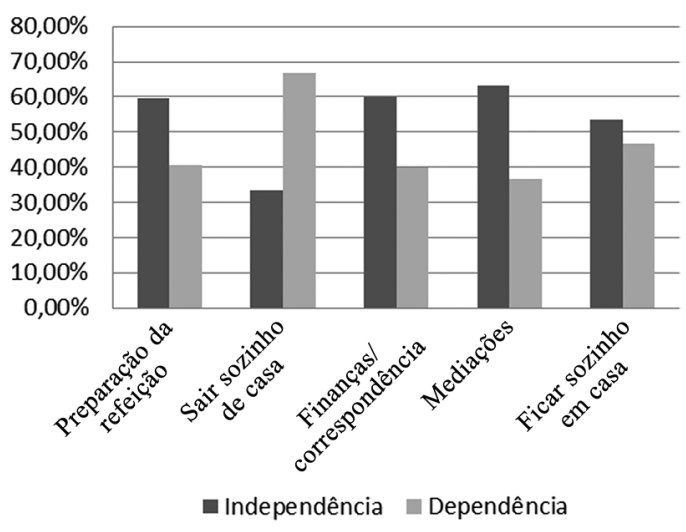

Figura 2. Grau de independência em cada uma das atividades não realizadas pelos idosos pela rotina da instituição, em porcentagem (\%). 


\section{Discussão}

Considerando-se os dados sociodemográficos da amostra, em relação ao gênero, 13 dos 15 idosos avaliados são mulheres, representando $86,66 \%$ do total. Esse resultado vai ao encontro do estudo de Camarano e Kanso (2011), que apontam que há predomínio de mulheres na população idosa, bem como entre idosos moradores de ILPI, compondo aproximadamente $57,6 \%$ dos residentes. No presente estudo, o índice de mulheres foi maior do que o do referido estudo, mas a amostra estudada é pequena.

A média de idade entre os idosos foi de 81,13 anos, sendo que $60 \%$ dos idosos avaliados estáo acima dos 80 anos. Davim et al. (2004) relataram que 30\% dos idosos residentes de ILPI na cidade de Natal-RN estavam acima de 80 anos e outro estudo, conduzido por Oliveira (2006), aponta que a média de idade dos idosos avaliados, em seu estudo, foi de 82 anos. Estes estudos relatam que a faixa etária representa aspecto importante a ser considerado na população idosa, pois, com o avanço da idade, maior é o risco de adoecimento e, consequentemente, um maior o grau de dependência.

Os resultados de desempenho na bateria DLOTCA-G indicam déficits cognitivos nos idosos. Converso e Iartelli (2007), em seu estudo, também encontraram idosos institucionalizados com déficits cognitivos, os quais representavam $76,72 \%$ dos idosos avaliados, mostrando o quão forte é a perda cognitiva nesta população.

O presente estudo, além de confirmar a presença de déficit cognitivo na população de idosos institucionalizados, aponta quais os domínios mais preservados - Percepção Espacial $(0,83 / 1)$ e Percepção Visual (3,26/4); e os mais comprometidos - Construção Visuomotora $(2,44 / 5)$ e Operaçóes de Pensamento (2,53/5).

As provas em que o desempenho foi mais baixo (Construção Visuomotora e Operaçóes de Pensamento) são mais complexas e exigem mais da capacidade cognitiva dos idosos, estando relacionadas ao funcionamento executivo.

Analisando-se o uso das mediaçóes durante a avaliação cognitiva, os idosos necessitaram do mais alto nível de mediação (nível 4 ou 5) em todos os domínios da bateria DLOTCA-G, o que também indica um alto grau de comprometimento cognitivo.

Em relação ao efeito da avaliação dinâmica, os idosos avaliados se beneficiaram do uso das mediaçóes, com melhora nas médias das pontuaçôes por domínio, nos domínios Percepção Visual (3,26 para 3,64 [1-4]), Percepção Espacial (0,83 para 0,98 [0-1]), Práxis
(1,55 para 1,80 [0-2]) e Construção Visuomotora (2,44 pra 3,11 [1-5]), sendo estes os que mais se beneficiaram com a mediação. Isto indica que, por mais que não tenham atingido a pontuação máxima, os idosos se beneficiaram do uso da mediação, melhorando seu desempenho.

Este é exatamente o objetivo da avaliação dinâmica, com o uso das mediaçôes: o de saber "como" e "o quê" melhora no desempenho do indivíduo ao receber um direcionamento. Essa é uma vantagem deste tipo de avaliação, pois tem foco nas variaçôes e mudanças que um indivíduo pode ter a partir do uso da mediação, possibilitando, entâo, o desenvolvimento de estratégias efetivas de remediação, além de mostrar que o aprendizado pode estar presente tanto no processo de avaliação como na vida do indivíduo (KATZ; AVERBUCH; EREZ, 2012).

A partir dos resultados da DAD, os idosos apresentaram 54\% de independência nas AVDs. As atividades que obtiveram maior porcentagem de independência estáo relacionadas às ABVDs, que são mais simples, como alimentar-se, e à continência. Por este motivo, tais atividades podem estar mais preservadas.

Já as atividades em que os idosos apresentaram menor independência fazem parte das AIVDs, como usar o telefone, fazer atividades fora de casa e atividades de lazer e de casa, que são mais complexas, podendo explicar o alto grau de dependência.

Os idosos apresentaram 66,27\% de independência nas ABVDs e $52,18 \%$ nas AIVDs. Desta maneira, as AIVDs estáo mais prejudicadas e, embora os idosos ainda apresentem certa independência em relação às AIVDs, é necessário um acompanhamento e estímulo da realização destas atividades. Para que isto ocorra, torna-se importante a avaliação constante da capacidade funcional dos idosos para criação de estratégias que minimizem a progressão das perdas funcionais e cognitivas. Observe-se que essas alteraçōes e déficits causados pelo declínio cognitivo e funcional acarretam diminuiçáo e/ou perdas nas habilidades dos idosos, interferindo de forma significativa na realização das AVDs, interferindo, então, em sua vida diária (DUARTE, 2003).

Os resultados apontaram correlaçấo estatisticamente significativa entre a cogniçáo e a funcionalidade nos idosos avaliados $(r=0,57 ; \mathrm{p}<0,05)$. A correlação é ainda mais significativa $(r=0,58 ; p<0,05)$ quando são consideradas as pontuaçôes pós-mediação, denotando, segundo Callegari-Jacques (2003), correlaçâo moderada. 
A correlação entre cognição e funcionalidade também foi encontrada no estudo de Converso e Iartelli (2007), no qual foi observada correlação de grau moderado entre o Índice de Barthel (capacidade funcional) e o Miniexame do Estado Mental (cognição), indicando que os estados mental e funcional possuem dependência estaticamente significativa $(r=0,44 ; \mathrm{p}<0,00)$.

Refletindo-se acerca de como a rotina institucional influencia o desempenho dos idosos em suas atividades, Pedretti e Early (2004) apontam que a desestruturaçáo ou modificação da rotina diária, como ocorre no contexto institucional - com a realização das tarefas de forma rotineira ou com a realização das atividades por outras pessoas sem ser os próprios idosos - gera uma disfunção ocupacional, levando à perda de habilidades que afetam negativamente os idosos. Isto foi observado na análise dos dados a partir do relato dos cuidadores, segundo a qual os idosos apresentariam 54\% de independência para realizar as atividades que não são permitidas pela rotina da instituição, exceto saírem sozinhos de casa.

Em ambientes institucionalizados, muitas vezes, o idoso não tem oportunidade de exercer sua autonomia, pois, pela rotina institucional, não decidem horários, como querem ficar, que tipos de atividades desejam fazer, entre outras situações que poderiam ser realizadas por eles, mesmo com auxílio. E assim, à luz do que afirmam Araújo e Ceolim (2007), o estímulo à autonomia e à independência do idoso institucionalizado deve ser a condição primordial para a manutenção da sua independência física e comportamental.

É importante ressaltar que, ao avaliar um indivíduo, é preciso escolher o instrumento adequado e adaptado para o foco da avaliaçáo.

Alguns testes são muito extensos ou complexos e, se existirem versóes mais breves, estas devem ser utilizadas. Sabe-se que os idosos se cansam mais rapidamente, o que pode gerar um pior desempenho na avaliação e que o resultado pode não corresponder à sua real capacidade. Para alguns idosos, várias sessóes curtas podem ser mais produtivas. Além disso, muitas provas podem gerar ansiedade; por isso, cabe ao profissional tentar minimizar essa ansiedade e orientar o idoso em relaçáo ao processo de avaliação. Se instalada, a ansiedade pode prejudicar severamente o desempenho do indivíduo (YASSUDA; ABREU, 2011).

Os aspectos supramencionados foram observados durante a coleta de dados e, pelo fato de a bateria DLOTCA-G ser bastante extensa e pelo nível de comprometimento cognitivo dos idosos, para $80 \%$ deles foram necessárias duas sessóes para que a avaliação fosse concluída, sendo que, para idosos normais, o tempo de aplicação do teste é, em média, de 45 minutos.

Outro aspecto observado foi o fato de que a agitação, a ansiedade e o nervosismo são determinantes para a realização dos testes, pois estes podem influenciar o desempenho do indivíduo. Houve perda amostral de três idosos, pois, para dois deles, sua agitação os impedia de interagir a ponto de focar-se no teste, e para um dos avaliados, sua ansiedade o fez desistir de continuar o processo de avaliação.

Algumas limitaçóes do estudo referem-se ao tamanho da amostra, que é pequeno, não sendo possível generalizar os dados; assim, mostram-se aplicáveis somente para os idosos desta amostra.

\section{Conclusão}

Os idosos avaliados apresentam déficits cognitivos principalmente em relaçáo às funções executivas, representadas pelas provas de Construção Visuomotora e Operaçóes de Pensamento. Ao receberem mediaçóes, o desempenho dos idosos melhora, indicando que eles se beneficiam com o uso destas.

Com relação à funcionalidade, observou-se que os idosos apresentaram 54\% de independência para a realização das AVDs, Sendo 66,27\% de independência nas ABVDs (principalmente alimentação e continência) e 52,18\% nas AIVDs (principalmente uso do telefone). Foram encontradas correlaçóes significativas moderadas entre a cogniçāo e a funcionalidade nos idosos avaliados.

Observou-se que a falta de autonomia e independência pode ocorrer por conta da rotina institucional, sendo, então, de suma importância uma maior reflexão acerca deste aspecto para que possam ser propostas açóes que visam a manter a capacidade funcional dos idosos institucionalizados pelo maior tempo possível.

Vale ressaltar que a avaliação cognitiva escolhida (DLOTCA-G) é um instrumento específico da Terapia Ocupacional, que possibilita ao profissional não somente a avaliação do nível cognitivo do idoso, mas também aponta qual é a estratégia mais adequada para auxiliar o idoso no desempenho das diversas atividades em seu dia a dia. Assim, DLOTCA-G é de grande valia para o profissional no momento da avaliação, bem como na orientação aos familiares e cuidadores. 


\section{Referências}

ARAÚJO, M. O. P. H.; CEOLIM, M. F. Avaliação do grau de independência de idosos residentes em instituiçóes de longa permanência. Revista da Escola de Enfermagem da USP, São Paulo, v. 41, n. 3, p. 378-385, 2007.

BAHIA, V. S. et al. Functional Disability in Alzheimer Disease: a validation study of the brazilian version of the Disability Assessment for Dementia (DAD-Br). Alzheimer Disease and Associated Disorders, Philadelfia, v. 24, n. 3, p. 291-295, 2010.

BARRETO, K. M. L.; TIRADO, M. G. Terapia Ocupacional. In: FREITAS, E. V. et al. Tratado de geriatria e gerontologia. Rio de Janeiro: Guanabara Koogan, 2002. p. 866-871.

BORN, T.; BOECHAT, N. S. A qualidade dos cuidados ao idoso institucionalizado. In: FREITAS, E. V. et al. Tratado de geriatria e gerontologia. Rio de Janeiro: Guanabara Koogan, 2011. p. 1299-1310.

CALLEGARI-JACQUES, S. M. Bioestatística: princípios e aplicaçóes. Porto Alegre: Artemed, 2003.

CAMARANO, A. A.; KANSO, S. As instituiçóes de longa permanência para idosos no Brasil. Revista Brasileira de Estudos de População, Rio de Janeiro, v. 27, n. 1, p. 233-235, 2010.

CAMARANO, A. A.; KANSO, S. Envelhecimento da população brasileira/uma contribuição demográfica. In: FREITAS, E. V. et al. Tratado de geriatria e gerontologia. Rio de Janeiro: Guanabara Koogan, 2011. p. 58-73.

CANINEU, P. R.; STELLA, F.; SAMARA, A. B. Transtorno cognitivo leve. In: FREITAS, E. V. et al. Tratado de geriatria e gerontologia. Rio de Janeiro: Guanabara Koogan, 2011. p. 169-177.

CONVERSO, M. E. R.; IARTELLI, I. Caracterizaçáo e análise do estado mental e funcional de idosos institucionalizados em instituiçóes públicas de longa permanência. Jornal Brasileiro de Psiquiatria, Rio de Janeiro, v. 56, n. 4, p. 267-272, 2007.

DAVIM, R. M. B. et al. Estudo com idosos de instituiçóes asilares no município de Natal/RN: características socioeconômicas e de saúde. Revista Latino-America de Enfermagem, Ribeiráo Preto, v. 12, n. 3, p. 518-524, 2004.

DUARTE, Y. A. O. Desempenho funcional e demandas assistenciais. In: LEBRÃO, M. L.; DUARTE, Y. A. O. O projeto SABE no município de São Paulo: uma abordagem inicial. São Paulo: OPAS, 2003. p. 185-200.

FARIAS, N.; BUCHALLA, C. M. A classificação internacional de funcionalidade, incapacidade e saúde da organização mundial da saúde: conceitos, usos e perspectivas. Revista Brasileira de Epidemiologia, São Paulo, v. 8, n. 2, p. 187-193, 2005.
GONTIJO, J. V. Estado cognitivo e funcional de idosos de uma Instituição de Longa Permanência do município de Divinópolis - MG. 2010. 65 f. Dissertação (Mestrado em Educação, Cultura e Organizaçóes Sociais) - Universidade do Estado de Minas Gerais, Divinópolis, 2010.

GRIEVE, J.; GNANASEKARAN, L. Neuropsicologia para terapeutas ocupacionais: cognição no desempenho ocupacional. São Paulo: Santos, 2010.

INSTITUTO BRASILEIRO DE GEOGRAFIA E ESTATÍSTICA - IBGE. Censo demográfico. Rio de Janeiro, 2010. Disponível em: <http://www.ibge.gov.br/home/ default.php>. Acesso em: 09 out. 2012.

KATZ, N.; AVERBUCH, S.; EREZ, A. B-H. Dynamic Lowenstein Occupational Therapy Cognitive Assessment-Geriatric Version (DLOTCA-G): assessing change in cognitive performance. The American Journal of Occupational Therapy: Official Publication of the American Occupational Therapy Association, Bethesda, v. 66, n. 3, p. 311-319, 2012. http://dx.doi.org/10.5014/ ajot.2012.002485. PMid:22549596.

KIELHOFNER, G.; BURKE, J. P.; IGI, C. H. Um modelo de ocupação humana - parte 4 avaliação e intervenção (modelo de tratamento, teoria). Revista de Terapia Ocupacional da USP, São Paulo, v. 2, n. 2-3, p. 127-144, 1991.

NEISTADT, M. E.; CREPEAU, E. B. Introdução à terapia ocupacional. In: NEISTAD, M. E.; CREPEAN, E. B. Willard \& Spackman: Terapia Ocupacional. Rio de Janeiro: Guanabara Koogan, 2002. p. 3-9.

NERI, A. L.; NERI, M. L. Envelhecimento cognitivo. In: FREITAS, E. V. et al. Tratado de geriatria e gerontologia. Rio de Janeiro: Guanabara Koogan, 2011. p. 14611476.

OLIVEIRA, S. F. D. Declinio cognitivo, funcionalidade e arranjos domiciliares entre os idosos do município de São Paulo. 2006. 109 f. Dissertação (Mestrado em Enfermagem) - Universidade de São Paulo, São Paulo, 2006.

PARENTE, M. A. M. et al. Cognição e envelhecimento. Porto Alegre: Artmed, 2006.

PEDRETTI, L. W.; EARLY, M. B. Desempenho ocupacional e modelos de prática para disfunção física. In: PEDRETTI, L. W.; EARLY, M. B. Terapia ocupacional: capacidades práticas para as disfunções físicas. São Paulo: Roca, 2004. p. 3 -13.

PERRACINI, M. R. Planejamento e adaptação do ambiente para pessoas idosas. In: FREITAS, E. V. et al. Tratado de geriatria e gerontologia. Rio de Janeiro: Guanabara Koogan, 2011. p. 1311-1323.

VAGETTI, G. C.; WEINHEIMER, M. S.; OLIVEIRA, V. Atendimento integral à saúde do idoso residente em instituição de longa permanência: uma experiência interdisciplinar. Estudos Interdisciplinar sobre o Envelhecimento, Porto Alegre, v. 11, p. 53-66, 2007. 
VIEIRA, E. B.; KOENIG, A. M. Avaliação cognitiva. In: FREITAS, E. V. et al. Tratado de Geriatria e Gerontologia. Rio de Janeiro: Guanabara Koogan, 2002. p. 921-928.
YASSUDA, M. S.; ABREU, V. P. S. Avaliação cognitiva do idoso. In: FREITAS, E. V. et al. Tratado de Geriatria e Gerontologia. Rio de Janeiro: Guanabara Koogan, 2011. p. 1486-1494.

\section{Contribuição dos Autores}

Renata Souza Mendes: contribuiu com a concepção e a redação do texto, organização de fontes e análises. Marcia Maria Pires Camargo Novelli: contribuiu com redação e revisão do texto. Todos os autores aprovaram a versão final do texto. 